\title{
Realization of a Single-Cooper-Pair Josephson Laser
}

\author{
Fei Chen, ${ }^{1}$ Juliang Li,${ }^{1}$ A. D. Armour, ${ }^{2}$ E. Brahimi,${ }^{1}$ Joel Stettenheim, ${ }^{1}$ \\ A. J. Sirois,${ }^{3}$ R. W. Simmonds,${ }^{4}$ M. P. Blencowe, ${ }^{1}$ and A. J. Rimberg ${ }^{1, *}$ \\ ${ }^{1}$ Department of Physics and Astronomy, \\ Dartmouth College, Hanover New Hampshire 03755, USA \\ ${ }^{2}$ School of Physics and Astronomy, University of Nottingham, \\ Nottingham NG7 2RD, United Kingdom \\ ${ }^{3}$ University of Colorado, 2000 Colorado Ave., Boulder, Colorado 80309, USA \\ ${ }^{4}$ National Institute of Standards and Technology, Boulder, Colorado 80305, USA
}

(Dated: June 26, 2014)

\begin{abstract}
We have embedded a voltage-biased Cooper pair transistor (CPT) in a high- $Q$ superconducting microwave cavity. When the energy given to a tunneling Cooper pair by the voltage bias is equal to a multiple of the cavity photon frequency, the cavity is pumped to a strongly non-equilibrium state. The cavity photons act back on the CPT, allowing us to enter a previously unstudied regime of strongly correlated electronic-photonic transport. We directly observe the effects of photonic backaction on Cooper pair transport, and see clear evidence for single-emitter lasing in the form of emission dominated by stimulated transport processes.
\end{abstract}

PACS numbers: 85.25.Cp,42.50.Ct,74.50.+r,73.23.Hk 
The quantum interaction of light with matter, long studied in the context of atomic systems $[1,2]$, has recently been extended to condensed matter systems through the advent of quantum optical experiments based on superconducting circuits [3]. Application of quantum optical techniques in this context has led to new regimes of strong coupling between light and matter [3], manipulation and readout of qubits [4, 5], generation of quantum states of light $[6,7]$ and development of ultra-low-noise quantum amplifiers [8-10]. A particularly familiar application of quantum optics is the laser, which in the single emitter regime has in atomic systems led to the production of pure photon number states [11] and sub-Poissonian photon statistics [12]. Analogous single-emitter superconducting devices have also been a topic of recent attention [13-17].

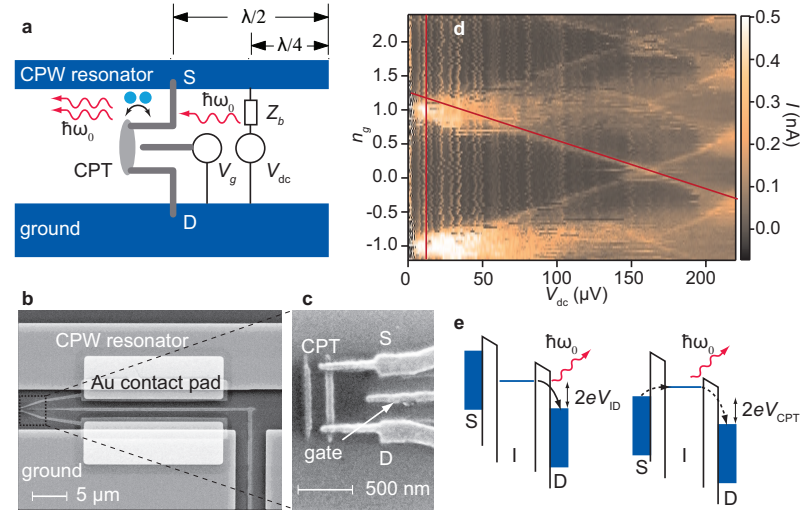

FIG. 1. (color online). (a) Schematic illustration of the circuit. (b) Electron micrograph of the CPT location. Proximitized $30 \mathrm{~nm}$ thick $\mathrm{Au} / \mathrm{Ti}$ contact pads provide dissipationless electrical contact between the CPT and the cavity, made of $100 \mathrm{~nm}$-thick Nb. The approximately $21.5 \mathrm{~mm}$-long cavity is coupled at either end via small capacitors to a waveguide with characteristic impedance $Z_{0}=50 \Omega$. (c) Micrograph of the $\mathrm{CPT}$ and its gate line. The CPT consists of a $7 \mathrm{~nm}$ thick superconducting $\mathrm{Al}$ island with charging energy $E_{c}=e^{2} / 2 C_{\Sigma}=h \times 8.7 \mathrm{GHz}$, where $C_{\Sigma}$ is the total island capacitance and coupled by small Josephson junctions to $70 \mathrm{~nm}$ thick Al leads. The Josephson coupling energy $E_{J}=h \times 17 \mathrm{GHz}$ is determined from the junction resistance. (d) CPT current $I_{\mathrm{CPT}}$ versus $V_{\mathrm{dc}}$ and $n_{g}$. (e) Sequential tunneling across the island-drain junction and co-tunneling across the CPT, both with simultaneous net photon emission. For the cotunneling process shown, the total CPT voltage $V_{\mathrm{CPT}}$ must satisfy $2 e V_{\mathrm{CPT}}=\hbar \omega_{0}$. For sequential tunneling the voltage across a single junction must satisfy a similar condition. 

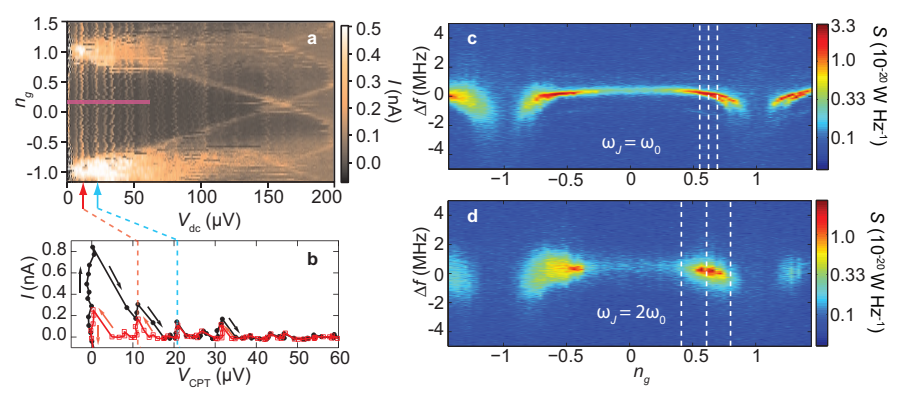

FIG. 2. (color online). (a) Current versus $V_{\mathrm{dc}}$ and $n_{g}$ showing the portions of the parameter space chosen for detailed investigation. (b) $I$ - $V$ characteristic for two sweep directions, as indicated, along the horizontal magenta line in (a). The vertical dashed red and blue lines indicate the locations of the first and second cotunneling features for $\omega_{J}=\omega_{0}$ and $\omega_{J}=2 \omega_{0}$ respectively. (c) and (d) Microwave spectral power density $S(\omega)$ of the cCPT over a $10 \mathrm{MHz}$ span versus detuning $\Delta f=f-f_{0}$ from the cavity resonant frequency $f_{0}=\omega_{0} / 2 \pi=5.256 \mathrm{GHz}$ for the first (c) and second (d) cotunneling peaks indicated by the red and blue arrows in (a). The vertical dashed lines in (c) and (d) indicate values of $n_{g}$ for which more detail is shown in Figs. 3 and 4.

Here we demonstrate a device consisting of a Cooper pair transistor [18] embedded in a high- $Q$ superconducting microwave cavity (cCPT) that acts as a single emitter laser and may offer a path toward simple, continual production of non-classical photons [19]. By applying a dc voltage to the CPT, we use the ac Josephson effect to inject photons into the cavity. Because the cavity $Q$ is large enough that the photon decay rate $\kappa$ is far smaller than the Cooper pair tunneling rate $\Gamma_{\mathrm{CP}}$, photonic backaction on tunneling cannot be neglected; $i$. e., stimulated rather than spontaneous tunneling events are dominant. The result is a new regime of simultaneous quantum coherent transport of Cooper pairs and microwave photons. This single-pair Josephson laser offers great potential for the production of amplitude-squeezed photon states and a rich environment for the study of the quantum dynamics of nonlinear systems [19-23].

The CPT is located at the voltage antinode at the center of a wavelength-long coplanar waveguide cavity with a resonant frequency $\omega_{0}=2 \pi \times 5.256 \mathrm{GHz}$ and quality factor $Q=$ $3.5 \times 10^{3}$ giving a photon decay rate $\kappa=\omega_{0} / Q=2 \pi \times 1.5 \mathrm{MHz}$. The cavity is modified by placement of dc bias lines at the voltage nodes located one quarter wavelength from either end of the cavity, as in Fig. 1a. These lines allow application of a dc voltage $V_{\text {dc }}$ to the central conductor of the cavity through a biasing impedance $Z_{b}$ without affecting the 
microwave properties of the cavity at its resonant frequency [24]. The CPT is fabricated with its source coupled to the central cavity conductor and its drain coupled to ground. A gate voltage $V_{g}$ is used to adjust the electrostatic potential of the CPT island via the gate charge $n_{g}=C_{g} V_{g} / e$, where $C_{g}$ is the gate capacitance. Escaping photons can be measured by microwave circuitry connected to the cavity's output port while the dc bias lines are used to probe electrical transport in the CPT [25].

The CPT is described by considering only two charge states, $|0\rangle$ and $|1\rangle$, corresponding to zero and one excess Cooper pair on the island. The states are separated by a gate-dependent electrostatic energy difference $2 \varepsilon=4 E_{c}\left(1-n_{g}\right)$, and are coupled via the Josephson energy $E_{J}$. By way of the ac Josephson effect, the dc bias gives rise to a characteristic drive frequency $\omega_{J}=2 e V_{\mathrm{CPT}} / \hbar$; here $V_{\mathrm{CPT}}$, the source-drain voltage at the CPT, is not generally equal to the applied voltage $V_{\mathrm{dc}}$. Introducing cavity photon annihilation and creation operators $a$ and $a^{\dagger}$, the cCPT Hamiltonian is given by

$$
H=\hbar \omega_{0} a^{\dagger} a+\varepsilon \sigma_{z}-E_{J} \sigma_{x} \cos \left[\varphi_{\mathrm{zp}}\left(a+a^{\dagger}\right)+\frac{1}{2} \omega_{J} t\right]
$$

where $\sigma_{x}$ and $\sigma_{z}$ are the Pauli matrices. In writing (1) we implicitly assume $4 E_{c} \gtrsim E_{J}$, justifying our truncation of the charge basis to two states. The first two terms in (1) describe the cavity photons and the CPT charge, respectively. The third term describes the coupling between the CPT and the cavity photons, and the effects of the voltage drive. In a standard CPT this latter term would read $E_{J} \sigma_{x} \cos \varphi / 2$ where $\varphi$, the total superconducting phase difference between the source and drain, is a classical variable [18]. Here, quantum fluctuations of the cavity photon field must be accounted for via the identification $\hat{\varphi} / 2=$ $\varphi_{\mathrm{zp}}\left(a+a^{\dagger}\right)$. The zeropoint phase parameter $\varphi_{\mathrm{zp}}=\sqrt{Z_{0} / 4 R_{Q}} \approx 0.04$, where $R_{Q}=h / 4 e^{2}=$ $6.45 \mathrm{k} \Omega$ is the resistance quantum, describes the strength of the quantum phase fluctuations of the cavity field $[22,25,26]$.

The CPT current $I_{\mathrm{CPT}}$ measured at $T=30 \mathrm{mK}$ versus $V_{\mathrm{dc}}$ and $n_{g}$ as in Fig. $1(\mathrm{~d})$ shows far richer behavior than in measurements of Cooper pair or single electron transistors coupled to lower $Q$ resonators [27, 28]. For $V_{\mathrm{dc}} \lesssim 150 \mu \mathrm{V}, I_{\mathrm{CPT}}$ is strictly $2 e$ periodic in $n_{g}$, with no indication of a tendency toward e-periodicity, suggesting that only Cooper pair transport is significant for low bias [29]. Two varieties of transport process due to interaction of the CPT with the cavity are observed. There is sequential tunneling [diagonal red line, Fig. 1(d)] for which tunneling of a Cooper pair through a junction results in net photon emission into the 

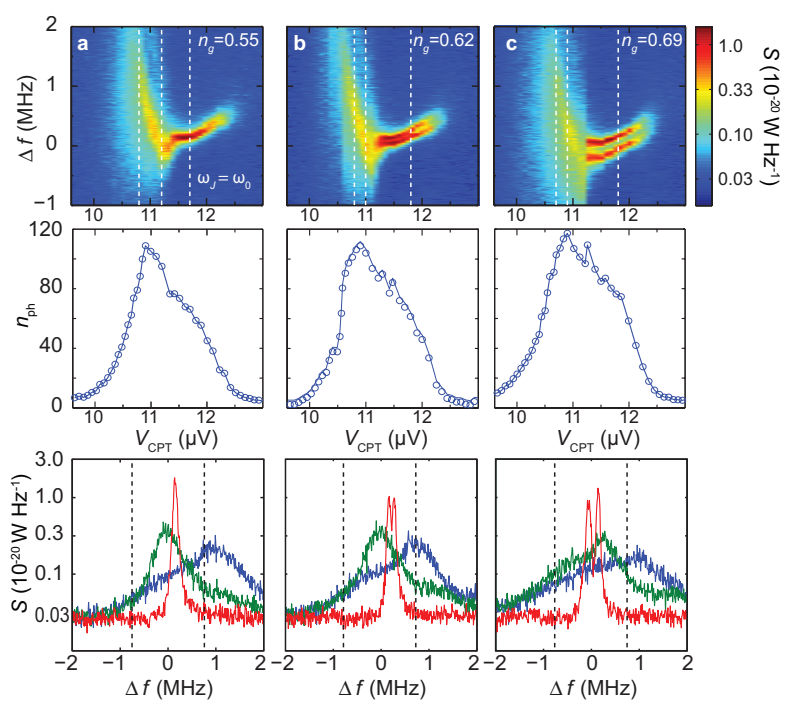

FIG. 3. (color online) (a)-(c) Top panels: emission or $\omega_{J} \approx \omega_{0}$ versus CPT voltage $V_{\mathrm{CPT}}$ and detuning $\Delta f$ for $n_{g}=0.55,0.62$ and 0.69 , as indicated by the white vertical dashed lines in Fig. 2(c). The bias voltage $V_{\mathrm{dc}}$ was swept from low to high in $75 \mathrm{nV}$ increments. Middle panels: measured $n_{\mathrm{ph}}$ versus $V_{\mathrm{CPT}}$ for each gate voltage. Bottom panels: individual spectra for each gate voltage measured at the values of $V_{\mathrm{CPT}}$ indicated by the dashed vertical lines in the top panels. Black vertical dashed lines indicate the nominal of bandwidth $f_{0} / Q$ of the cavity. Specific values of $V_{\mathrm{CPT}}$ are: (a) $10.8 \mu \mathrm{V}$ (blue), $11.2 \mu \mathrm{V}$ (green), and $11.7 \mu \mathrm{V}$ (red); (b) $10.8 \mu \mathrm{V}$ (blue), $11.0 \mu \mathrm{V}$ (green), and $11.8 \mu \mathrm{V}$ (red); (c) $10.7 \mu \mathrm{V}$ (blue), $10.9 \mu \mathrm{V}$ (green), and $11.8 \mu \mathrm{V}$ (red).

cavity [left panel, Fig. 1(e)]. There is also cotunneling [vertical red line, Fig. 1(d)], in which a Cooper pair is transferred from source to drain through an energetically forbidden state [right panel of Fig. 1(e))] again with net photon emission. In the presence of large numbers $n_{\mathrm{ph}}$ of cavity photons, both processes can be strongly affected by the cavity field [Fig. 1(a)].

Here we focus on the first two cotunneling features as indicated in Fig. 2(a), at $V_{\mathrm{CPT}} \approx$ $11 \pm 1$ and $22 \pm 1 \mu \mathrm{V}$, corresponding to drive frequencies $\omega_{J} \approx \omega_{0}$ and $2 \omega_{0}$ respectively and to net emission of one or two cavity photons. The uncertainty in $V_{\mathrm{CPT}}$ is due to the $\sim \pm 1 \mu \mathrm{V}$ peak-to-peak noise of our de voltage amplifier. Detailed behavior of $I_{\mathrm{CPT}}$ versus $V_{\mathrm{CPT}}$ is shown in Fig. 2(b) for $n_{g}$ far from charge degeneracy at $n_{g}= \pm 1$. The current is hysteretic in $V_{\mathrm{CPT}}$, and has sharp steps at fixed voltages $V_{\mathrm{CPT}}$ corresponding to the cotunneling features (see also Ref. 25). The voltage width of the steps, while not resolved, is clearly small 
compared to the voltages at which they occur, a consequence of the high cavity $Q$. Similar $I-V$ characteristics in the phenomena of Shapiro steps [30] and Fiske modes [31] indicate frequency matching between Josephson oscillations and an oscillatory electromagnetic field. In our context, such a field can only arise from photons injected into the cavity by tunneling Cooper pairs. From the maximum measured current (over all $n_{g}$ ), which is $I_{\mathrm{CPT}} \approx 300 \mathrm{pA}$ at both $V_{\mathrm{CPT}} \approx 11 \mu \mathrm{V}$ and $22 \mu \mathrm{V}$, we find $\Gamma_{\mathrm{CP}}=I_{\mathrm{CPT}} / 2 e \approx 9 \times 10^{8} \mathrm{~s}^{-1} \gg \kappa$, corresponding to the very high $Q$ regime. Furthermore, we estimate the steady state number of cavity photons $n_{\mathrm{ph}}=I_{\mathrm{CPT}} V_{\mathrm{CPT}} / \kappa \hbar \omega_{0} \approx 100(200)$ at $\omega_{J}=\omega_{0}\left(2 \omega_{0}\right)$, as verified by microwave measurements below.

Note that $n_{\mathrm{ph}}$ is far larger than the thermal photon number $n_{\mathrm{th}}=1 /\left(e^{\hbar \omega_{0} / k_{B} T}-1\right) \approx$ $6 \times 10^{-3}$ for an estimated electron temperature $T \approx 50 \mathrm{mK}$. From the estimated cavity photon number $n_{\mathrm{ph}}$ and the sharp steps in the $I-V$ characteristic, two important conclusions can now be drawn: first, the cavity photon state is very far from equilibrium; and furthermore we are in the high $Q$ regime for which cavity photons can significantly affect Cooper pair tunneling. For comparison, junctions $[32,33]$ or CPTs [27, 28] coupled to low- $Q$ cavities satisfy $\Gamma_{\mathrm{CP}} \ll \kappa$. In that case $n_{\mathrm{ph}}$ remains low, allowing a thermal description of the cavity photons, and resonances in the $I-V$ characteristics are broad, with a width only slightly smaller than the resonant voltage.

To verify photon emission at the current steps, we measure the emitted microwave spectral power density $S(\omega)$ versus $n_{g}$ for $\omega_{J}=\omega_{0}$ and $2 \omega_{0}$. The microwave amplifier chain was calibrated using the shot noise of the CPT when biased on its quasiparticle branch. Also, to minimize jitter in $\omega_{J}$ we used low-noise bias circuitry that greatly improved emission stability at the cost of reduced precision in current measurements [25]. For both $\omega_{J}=\omega_{0}$ and $2 \omega_{0}$ there is strong emission close to $\omega_{0}$ as can be seen in Fig. 2 (c) and (d). As with the CPT current, both emission patterns are strictly $2 e$-periodic. In both cases, the emission dies out near charge degeneracy at $n_{g}= \pm 1$.

In Figs. 3 and 4 we show emission spectra $S(\omega)$ and cavity photon number $n_{\text {ph }}$ versus $V_{\mathrm{CPT}}$ at $\omega_{J}=\omega_{0}$ and $2 \omega_{0}$ for representative values of $n_{g}$, as well as spectra at particular values of $V_{\mathrm{CPT}}$. Here $n_{\mathrm{ph}}$ was deduced from the integrated cCPT microwave output power $P_{\mathrm{CPT}}$ through the relation $n_{\mathrm{ph}}=P_{\mathrm{CPT}} / \kappa \hbar \omega_{0}$. The values of $V_{\mathrm{CPT}}$ used in Figs. 3 and 4 were derived from $P_{\mathrm{CPT}}$ and knowledge of the bias circuitry [25]. For $\omega_{J}=\omega_{0}$ in Fig. 3 we see that as expected for a single-atom emitter there is no lasing threshold, with the 
cavity occupation $n_{\mathrm{ph}}$ climbing smoothly from zero as $V_{\mathrm{CPT}}$ is increased. For $V_{\mathrm{CPT}} \lesssim 11 \mu \mathrm{V}$ the emission linewidth of roughly $1 \mathrm{MHz}$ shows clear narrowing over the intrinsic cavity linewidth $\kappa=2 \pi \times 1.5 \mathrm{MHz}$. At $V_{\mathrm{CPT}} \approx 11 \mu \mathrm{V}$, there is a sharp change in the emission: the linewidth drops by roughly an order of magnitude, to as low as $70 \mathrm{kHz}$, slightly larger than the residual jitter in $\omega_{J} / 2 \pi$ of about $35 \mathrm{kHz}$ [25]. Over the same range in $V_{\mathrm{CPT}}$ the cavity photon occupancy $n_{\mathrm{ph}}$ reaches a maximum value on the order of 100 , verifying our estimates from the $I-V$ characteristics.

For $\omega_{J}=2 \omega_{0}$ in Fig. 4 there is again no clear sign of a threshold as $V_{\mathrm{CPT}}$ is increased. There is no sudden sharpening of the spectrum in this case; instead, the emission simply cuts off abruptly for $V_{\mathrm{dc}} \gtrsim 22 \mu \mathrm{V}$, just after the cavity reaches its maximum occupancy of roughly $n_{\mathrm{ph}} \approx 200$, again in excellent agreement with the $I$ - $V$ characteristics. The minimum linewidth of the emission spectra is roughly $350 \mathrm{kHz}$ [25], significantly below the bare cavity linewidth. In Fig. 3, as charge degeneracy is approached for $\omega_{J}=\omega_{0}$, the spectrum splits into two narrowly separated peaks at around $n_{g}=0.62$. The separation of these peaks increases as $n_{g}$ approaches charge degeneracy, accompanied by a shift in the emission frequency toward negative detuning. The spectra in Fig. 4 also exhibit a clear though less pronounced pulling toward negative detuning. The origin of the splitting in Fig. 3 is unclear. One possibility is quasiparticle poisoning of the CPT island $[18,29]$ for which even and odd charge states of the island might give rise to slightly different emission frequencies. The island would then have a significant probability of being in an odd charge state, however, and we would expect emission to occur at $n_{g}= \pm 1$, corresponding to an $e$-shifted version of the emission at $n_{g}=0$. While such poisoning cannot be ruled out, the absence of emission at $n_{g}= \pm 1$ makes it seem unlikely. Other possibilities include the simultaneous existence of two different phases for the cavity oscillations [19], each with slightly different emission frequency, or the influence of a higher CPT energy band, which draws close to the CPT ground state at charge degeneracy [34]. Further modeling is required to answer this question.

The standard theoretical approach describing transport in the CPT is so-called environmental $P(E)$ theory $[35,36]$, which describes emission or absorption of photons by tunneling Cooper pairs to or from an environment with a frequency-dependent impedance $Z_{t}(\omega)$. As confirmed by recent experiments on single junctions [33], if the impedance $Z_{t}(\omega)$ is peaked at a frequency $\omega_{0}$ (as for a resonance) there is substantial probability $P(E)$ of photon emission when the junction is biased at a voltage given by $V=\hbar \omega_{0} / 2 e$. Near that voltage there is 
a smooth current peak due to incoherent tunneling of Cooper pairs [23, 32, 33] that tracks the shape of $Z_{t}(\omega)$.

A key assumption of $P(E)$ theory is that the environment is in thermal equilibrium. At low temperature (10s of $\mathrm{mK}$ ) and high frequency (several $\mathrm{GHz}$ ) the thermal occupation of environmental modes is small $\left(n_{\text {th }} \ll 1\right)$; processes described by $P(E)$ are spontaneous, not stimulated. In the low- $Q$ regime, tunneling rates $\Gamma_{\mathrm{CP}}$ predicted by environmental theory satisfy $\Gamma_{\mathrm{CP}} \ll \kappa$, so that the steady-state cavity photon occupation also satisfies $n_{\mathrm{ph}}=\Gamma_{\mathrm{CP}} / \kappa \ll 1$ : for low $Q, P(E)$ theory is internally consistent. On the other hand, in the high- $Q$ regime of the Josephson laser, straightforward application of environmental theory fails. Treating the CPT at the cotunneling features as a gate-tunable single junction $[25,37]$, the theory predicts an incoherent cotunneling rate $\Gamma_{\cot }^{(1)}$ for $\omega_{J}=\omega_{0}$ such that $n_{\mathrm{ph}}=\Gamma_{\mathrm{cot}}^{(1)} / \kappa \sim 4 Q^{2} \varphi_{\mathrm{zp}}^{2} \sim 10^{5}[25] . P(E)$ theory therefore predicts $n_{\mathrm{ph}} \gg 1$, violating its assumption of an equilibrium environment: in the high- $Q$ regime, $P(E)$ theory is internally inconsistent. Unsurprisingly, specific predictions of environmental theory for tunneling rates, cavity occupation, and spectral width fail in our case [25].

The failure of $P(E)$ theory to accurately describe the Josephson laser arises from its neglect of backaction by long-lived cavity photons on emission. If $n_{\mathrm{ph}}$ satisfies $\varphi_{\mathrm{zp}} n_{\mathrm{ph}}^{1 / 2} \gtrsim 1$, so that the full nonlinearity of the Hamiltonian (1) is important, the steady state values of $\Gamma_{\mathrm{CP}}$ and $n_{\mathrm{ph}}$ will be dominated by photon-mediated (stimulated) emission or absorption processes, rather than the spontaneous processes described by $P(E)$ theory. One also expects much narrower linewidths than those predicted by environmental theory for oscillating states of the cavity, with the residual jitter in $\omega_{J}$ setting a lower limit.

To account for the effects of the stimulated processes inherent in (1), we use a semiclassical approximation [25] that allows us to compute the expectation value $\langle a\rangle$ in the long-time limit. Writing $\langle a\rangle=\tilde{\alpha} e^{-i \omega_{0} t}$, we find that

$$
\begin{aligned}
\dot{\tilde{\alpha}}= & -\frac{\tilde{\alpha}}{2 Q}-i \frac{E_{J} \varphi_{\mathrm{zp}}}{\hbar} \mathrm{e}^{i \omega_{0} t}\left(\sigma+\sigma^{*}\right) \\
& \times \sin \left[\varphi_{\mathrm{zp}}\left(\tilde{\alpha} \mathrm{e}^{-i \omega_{0} t}+\tilde{\alpha}^{*} \mathrm{e}^{i \omega_{0} t}\right)+\frac{1}{2} \omega_{J} t\right],
\end{aligned}
$$

with $\sigma=\left\langle\sigma_{+}\right\rangle$where $\sigma_{+}=|1\rangle\langle 0|$. Resonances occur when the damping term $(-\tilde{\alpha} / 2 Q)$ is matched by another term with no overall time dependence. To see when this happens, we can initially neglect the effect of the cavity on the behavior of $\sigma$. In this limit, the CPT island charge at long times is a periodic function of the drive frequency $\sigma=\left\langle\sigma_{+}\right\rangle=$ 


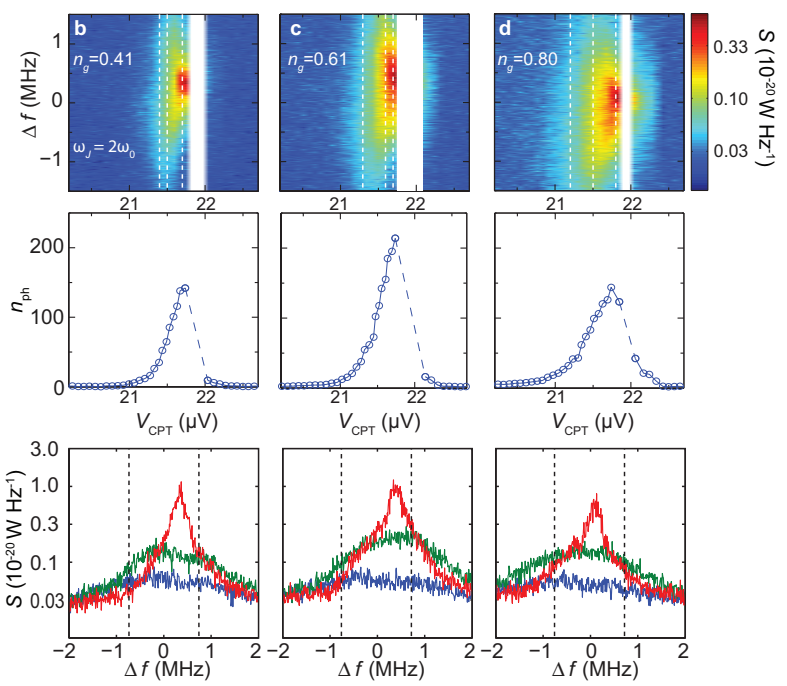

FIG. 4. (color online) (a)-(c) Top panels: emission for $\omega_{J} \approx 2 \omega_{0}$ versus CPT voltage $V_{\mathrm{CPT}}$ for $n_{g}=0.41,0.61$ and 0.80, as indicated by the vertical dashed lines in Fig. 2(d). The bias voltage $V_{\mathrm{dc}}$ was swept from low to high in $75 \mathrm{nV}$ increments. The gaps in the spectra (white) correspond to discontinuities in $V_{\mathrm{CPT}}$. Middle panels: measured $n_{\mathrm{ph}}$ versus $V_{\mathrm{CPT}}$ for each gate voltage. Bottom panels: individual spectra for each gate voltage measured at the values of $V_{\mathrm{CPT}}$ indicated by the white dashed lines in the top panels. Black vertical dashed lines indicate the nominal of bandwidth $f_{0} / Q$ of the cavity. Specific values of $V_{\mathrm{CPT}}$ are: (a) $21.4 \mu \mathrm{V}$ (blue), $21.5 \mu \mathrm{V}$ (green), and $21.7 \mu \mathrm{V}$ (red); (b) $21.3 \mu \mathrm{V}$ (blue), $21.6 \mu \mathrm{V}$ (green), and $21.7 \mu \mathrm{V}$ (red); (c) $21.2 \mu \mathrm{V}$ (blue), $21.5 \mu \mathrm{V}$ (green), and $21.8 \mu \mathrm{V}$ (red).

$\sum_{n}^{\prime} c_{n} \mathrm{e}^{i n \omega_{J} t / 2}$, where for reasons of symmetry [38], the sum runs over odd integers (indicated by the prime). Expanding the sinusoidal term, the most general resonance condition is $k \omega_{0}=p \omega_{J}$, corresponding to $k$ photons being produced by the cotunneling of $p$ Cooper pairs. To determine $n_{\mathrm{ph}} \simeq|\tilde{\alpha}|^{2}$, we need to include the effect of $\tilde{\alpha}$ on $\sigma$ and so integrate the full set of semiclassical equations of motion derived from (1). For our experimental parameters we obtain $n_{\mathrm{ph}} \approx 120$ for $\omega_{J}=\omega_{0}$ and $n_{\mathrm{ph}} \approx 250$ for $\omega_{J}=2 \omega_{0}$. In contrast with $P(E)$ theory, the full semiclassical treatment of the cCPT, including the backaction of cavity photons, correctly predicts $n_{\mathrm{ph}}$ for both $\omega_{J}=\omega_{0}$ and $2 \omega_{0}$. It also predicts that emission should vanish at the charge degeneracy points, where the CPT island is effectively decoupled from the cavity [25]. Finally, the oscillatory cavity state predicted by the model [25] is consistent with the narrow emission patterns in Fig. 3 and 4, and the Shapiro-like $I-V$ characteristics in Fig. 2(b). 
When evaluating lasing in single-emitter devices such as the cCPT, care must be taken, since many of the criteria familiar from multi-emitter lasers, $e . g$. the existence of a threshold and changes in photon statistics no longer clearly apply [12]. Here, following the definition of the term, we say that lasing occurs when stimulated processes dominate over spontaneous ones [39]. Since the cavity photon occupancy $n_{\mathrm{ph}}$ cannot be correctly predicted without the inclusion of stimulated processes, this criterion is clearly met in the cCPT. We thus argue that it must be considered a laser.

In conclusion, we have demonstrated lasing by means of a new quantum coherent transport process involving the interaction of Cooper pairs and photons. We have provided clear evidence of stimulated transport processes, and have shown good agreement between our results and a semiclassical theory. Fully quantum calculations on a simpler single-junction model $[19,25]$ predict that the photons generated by the cCPT are likely strongly amplitude squeezed. Experimental verification of this prediction is a clear direction for future work. The single-Cooper-pair Josephson laser may ultimately serve as a convenient, easy-to-use source of amplitude squeezed light, and could form the basis of a new class of electrical or photonic amplifiers. It could also serve as an important platform for the study of the quantum dynamics of strongly nonlinear systems.

This work was supported by the NSF under grants DMR-1104790 and DMR-1104821, by AFOSR/DARPA under agreement FA8750-12-2-0339, and by EPSRC (U.K.) under Grant No. EP/I017828/1.

* ajrimberg@dartmouth.edu

[1] D. F. Walls and G. J. Milburn, Quantum Optics (Springer, Berlin, 2008).

[2] S. Haroche and J.-M. Raimond, Exploring the Quantum (Oxford University Press, Oxford, 2006).

[3] A. Wallraff, D. I. Schuster, A. Blais, L. Frunzio, R.-S. Huang, J. Majer, S. Kumar, S. M. Girvin, and R. J. Schoelkopf, Nature 431, 162 (2004).

[4] L. DiCarlo, M. D. Reed, L. Sun, B. R. Johnson, J. M. Chow, J. M. Gambetta, L. Frunzio, S. M. Girvin, M. H. Devoret, and R. J. Schoelkopf, Nature 467, 574 (2010).

[5] M. D. Reed, L. DiCarlo, B. R. Johnson, L. Sun, D. I. Schuster, L. Frunzio, and R. J. 
Schoelkopf, Phys. Rev. Lett. 105, 173601 (2010).

[6] M. Hofheinz, H.Wang, M. Ansmann, R. C. Bialczak, E. Lucero, M. Neeley, A. D. O'Connell, D. Sank, J. Wenner, J. M. Martinis, and A. N. Cleland, Nature 459, 546 (2009).

[7] G. Kirchmair, B. Vlastakis, Z. Leghtas, S. E. Nigg, H. Paik, E. Ginossar, M. Mirrahimi, L. Frunzio, S. M. Girvin, and R. J. Schoelkopf, Nature 495, 205 (2013).

[8] I. Siddiqi, R. Vijay, F. Pierre, C. M. Wilson, M. Metcalfe, C. Rigetti, L. Frunzio, and M. H. Devoret, Phys. Rev. Lett. 93, 207002 (2004).

[9] M. A. Castellanos-Beltran, K. D. Irwin, G. C. Hilton, L. R. Vale, and K. W. Lehnert, Nature Phys. 4, 928 (2008).

[10] N. Bergeal, F. Schackert, M. Metcalf, R. Vijay, V. E. Manucharyan, L. Frunzio, D. E. Prober, R. J. Schoelkopf, S. M. Girvin, and M. H. Devoret, Nature 465, 64 (2010).

[11] B. T. H. Varcoe, S. Brattke, M. Weidinger, and H. Walther, Nature 403, 743 (2000).

[12] J. McKeever, A. Boca, A. D. Boozer, J. R. Buck, and H. J. Kimble, Nature 425, 268 (2003).

[13] D. A. Rodrigues, J. Imbers, and A. D. Armour, Phys. Rev. Lett. 98, 067204 (2007).

[14] O. Astafiev, K. Inomata, A. O. Niskanen, T. Yamamoto, Yu. A. Pashkin, Y. Nakamura, and J. S. Tsai, Nature 449, 588 (2007).

[15] J. Q. You, Y.-X. Liu, C. P. Sun, and F. Nori, Phys. Rev. B 75, 104516 (2007).

[16] J. Hauss, A. Fedorov, C. Hutter, A. Shnirman, and G. Schön, Phys. Rev. Lett. 100, 037003 (2008).

[17] S. Ashhab, J. R. Johansson, A. M. Zagoskin, and F. Nori, New J. Phys. 11, 023030 (2009).

[18] P. Joyez, P. Lafarge, A. Filipe, D. Esteve, and M. H. Devoret, Phys. Rev. Lett. 72, 2458 (1994).

[19] A. D. Armour, M. P. Blencowe, E. Brahimi, and A. J. Rimberg, Phys. Rev. Lett. 111, 247001 (2013).

[20] S. Habib, K. Shizume, and W. H. Zurek, Phys. Rev. Lett. 80, 4361 (1998).

[21] S. Chaudhury, A. Smith, B. E. Anderson, S. Ghose, and P. S. Jessen, Nature 461, 768 (2009).

[22] M. P. Blencowe, A. J. Rimberg, and A. D. Armour, in Fluctuating Nonlinear Oscillators, edited by M. Dykman (Oxford University Press, Oxford, 2012) pp. 33-58.

[23] V. Gramich, B. Kubala, S. Rohrer, and J. Ankerhold, Phys. Rev. Lett. 111, 247002 (2013).

[24] F. Chen, A. J. Sirois, R. W. Simmonds, and A. J. Rimberg, Appl. Phys. Lett. 98, 132509 (2011). 
[25] See the Supplementary Material for details of the voltage biasing scheme and microwave measurements, derivation of the cCPT Hamiltonian, and discussion of $P(E)$ theory and the semiclassical approximation for the cCPT dynamics.

[26] M. Marthaler, J. Leppäkangas, and J. H. Cole, Phys. Rev. B 83, 180505(R) (2011).

[27] W. Lu, K. D. Maranowski, and A. J. Rimberg, Phys. Rev. B 65, 060501 (2002).

[28] Yu. A. Pashkin, H. Im, J. Leppäkangas, T. F. Li, O. Astafiev, A. A. Abdumalikov, E. Thuneberg, and J. S. Tsai, Phys. Rev. B 83, 020502(R) (2011).

[29] A. Amar, D. Song, C. J. Lobb, and F. C. Wellstood, Phys. Rev. Lett. 72, 3234 (1994).

[30] S. Shapiro, Phys. Rev. Lett. 11, 80 (1963).

[31] M. D. Fiske, Rev. Mod. Phys. 36, 221 (1964).

[32] T. Holst, D. Esteve, C. Urbina, and M. H. Devoret, Phys. Rev. Lett. 73, 3455 (1994).

[33] M. Hofheinz, F. Portier, Q. Baudouin, P. Joyez, D. Vion, P. Bertet, P. Roche, and D. Esteve, Phys. Rev. Lett. 106, 217005 (2011).

[34] D. J. Flees, S. Han, and J. E. Lukens, Phys. Rev. Lett. 78, 4817 (1997).

[35] M. H. Devoret, D. Esteve, H. Grabert, G.-L. Ingold, H. Pothier, and C. Urbina, Phys. Rev. Lett. 64, 1824 (1990).

[36] S. M. Girvin, L. I. Glazman, M. Jonson, D. R. Penn, and M. D. Stiles, Phys. Rev. Lett. 64, $3183(1990)$.

[37] S. V. Lotkhov, S. A. Bogoslovsky, A. B. Zorin, and J. Niemeyer, Phys. Rev. Lett. 91, 197002 (2003).

[38] M. Grifoni and P. Hänggi, Phys. Reports 204, 229 (1998).

[39] Y. Mu and C. M. Savage, Phys. Rev. A 46, 5944 (1992). 